\title{
Operative intracranial infection following craniotomy
}

\author{
Shervin R. Dashti, M.D., Ph.D., Humain Baharvahdat, M.D., \\ Robert F. Spetzler, M.D., Eric Sauvageau, M.D., Steven W. Chang, M.D., \\ Michael F. Stiefel, M.D., Min S. Park, M.D., and Nicholas C. Bambakidis, M.D.
}

\author{
Division of Neurological Surgery, Barrow Neurological Institute, St. Joseph's Hospital and Medical \\ Center, Phoenix, Arizona
}

\begin{abstract}
Object. Postoperative infection after cranial surgery is a serious complication that requires immediate recognition and treatment. In certain cases such as postoperative meningitis, the patient can be treated with antibiotics only. In cases that involve a bone flap infection, subdural empyema, or cerebral abscess, however, reoperation is often needed. There has been significant disagreement regarding the incidence of postoperative central nervous system (CNS) infections following cranial surgery. In this paper the authors' goal was to perform a retrospective review of the incidence of CNS infection after cranial surgery at their institution. They focused their review on those patients who required repeated surgery to treat the infection.

Methods. The authors reviewed the medical records and imaging studies in all patients who underwent a craniotomy or stereotactic drainage for CNS infection over the past 10 years. Subgroup analysis was then performed in patients whose infection was a result of a previous cranial operation to determine the incidence, factors associated with infection, and the type of infectious organism. Patients treated nonoperatively (that is, those who received intravenous antibiotics for postoperative meningitis or cellulitis) were not included. Patients treated for wound infection without intracranial pus were also not included.

Results. During the study period from January 1997 through December 2007, 16,540 cranial surgeries were performed by 25 neurosurgeons. These included elective as well as emergency and trauma cases. Of these cases 82 $(0.5 \%)$ were performed to treat postoperative infection in 50 patients. All 50 patients underwent their original surgery at the authors' institution. The median age was 51 years (range 2-74 years). There were 26 male and 24 female patients.

The most common offending organism was methicillin-sensitive Staphylococcus aureus, which was found in 10 of 50 patients. Gram-negative rods were found in 15 patients. Multiple organisms were identified in specimens obtained in 5 patients. Six patients had negative cultures. Most craniotomies leading to subsequent infection were performed for tumors or other mass lesions (23 of 50 patients), followed by craniotomies for hemorrhage and vascular lesions. Almost half of the patients underwent $>1$ cranial operation before presenting with infection.

Conclusions. Postoperative infection after cranial surgery is an important phenomenon that needs immediate recognition. Even with strict adherence to sterile techniques and administration of antibiotic prophylaxis, a small percentage of these patients will develop an infection severe enough to require reoperation.
\end{abstract}

(DOI: 10.3171/FOC/2008/24/6/E10)

\section{KEY WORDS - brain abscess - postoperative cranial infection - subdural empyema}

$\mathrm{P}$ OSTOPERATIVE infection following cranial surgery is a serious complication that requires immediate recognition and treatment. After neurosurgical procedures, infection most commonly presents as meningitis, subdural empyema, or cerebral abscess. ${ }^{6,9,14,17,21}$ Although meningitis can occur after any type of cranial surgery, it is most common after approaches to the posterior fossa and is often associated with CSF leak. A high index of suspicion can lead to prompt investigation, including lumbar puncture for CSF studies. Once diagnosed, postoperative meningitis can often be treated with a course of intravenous antibiotics

Abbreviation used in this paper: $\mathrm{CSF}=$ cerebrospinal fluid. and/or steroids. ${ }^{3,13,16}$ In contrast, cases that involve a bone flap infection, subdural empyema, or cerebral abscess usually require a repeated operation. . $^{6,911,12,14,21}$ Infected bone flaps are usually removed and discarded. The goal of the surgery is then to evacuate pus and infected debris as much as possible to allow the intravenous antibiotics to work effectively. Sometimes multiple operations are required for reaccumulation of subdural or intraparenchymal pus.

A $0.8-7 \%$ incidence of postoperative infection has been reported in large series of neurosurgical procedures in which patients received preoperative antibiotic prophylaxis. ${ }^{1,5,9,15,19}$ Several retrospective as well as randomized trials have shown that, without antibiotic prophylaxis, the infection rate is significantly higher $(>10 \%))^{4,5,10,18,20,22-24}$ Most 
S. R. Dashti et al.

TABLE 1

Presenting symptoms in 50 patients with intracranial infections

\begin{tabular}{lc}
\hline \multicolumn{1}{c}{ Symptoms } & No. of Patients $(\%)$ \\
\hline fever & $11(22)$ \\
purulent drainage & $17(34)$ \\
mental status change & $18(36)$ \\
headache & $10(20)$ \\
swelling & $7(14)$ \\
seizure & $2(4)$ \\
\hline
\end{tabular}

of these studies included all postoperative infections, both surgical and medical, including nonoperative cases. Given the wide range of incidence of postoperative infection reported in the literature, we reviewed our experience in an extremely high volume center. We were interested in more definitively determining the rate of postcraniotomy infections that required reoperation. We retrospectively reviewed the cases of postoperative infection after cranial surgery over the past 10 years. We then focused on those patients who required a reoperation to control the infection. Patients who underwent simple wound revision without pus evacuation were not included.

\section{Clinical Materials and Methods}

The medical records and imaging studies of all patients who underwent craniotomy at the Barrow Neurological Institute in Phoenix, Arizona, for a central nervous system infection over the past 10 years were reviewed. Subgroup analysis was then performed in patients whose infection was a result of a previous cranial operation to determine the incidence, factors associated with infection, and the type of infectious organism. Patients with wound infection, bone flap infection, epidural abscess, subdural abscess, and cerebral abscess were included in this series. Patients who were treated nonoperatively with antibiotics for postoperative meningitis or cellulitis were excluded. Patients who underwent simple wound revision without pus evacuation were also excluded.

The patients' previous cranial operations included both elective and emergency procedures. The emergency cases included cases of trauma, given that Barrow Neurological Institute is based at St. Joseph's Hospital in Phoenix, which is a Level 1 trauma center. Patients whose original cranial surgery was performed at another institution were excluded from the study.

All patients received $1 \mathrm{~g}$ of a first-generation cephalosporin $<1$ hour before incision. In the majority of cases, this was followed by a 24-hour postoperative course of antibiotics. In more recent years, however, most of the operations were performed after administration of the preoperative antibiotic dose only. All surgical sites were shaved using an electric razor. The surgical preparation consisted of 5 minutes of scrubbing with Betadine scrub solution, followed by painting with Betadine paint solution. For patients with an iodine allergy, the scrub and paint were done using chlorohexidine solution. In most cases, Ioban (3M) antibacterial film was applied to the scalp prior to incision.
TABLE 2

Postoperative infections

\begin{tabular}{lc}
\hline \hline \multicolumn{1}{c}{ Type of Infection } & No. of Patients $(\%)$ \\
\hline wound infection & $26(52)$ \\
subdural empyema & $7(14)$ \\
epidural empyema & $27(54)$ \\
cerebral abscess & $8(16)$ \\
posterior fossa abscess & $2(4)$ \\
bone flap infection & $22(44)$ \\
\hline
\end{tabular}

\section{Statistical Analysis}

The chi-square test was used to compare the difference between proportions for $\geq 2$ groups. The nonparametric Mann-Whitney U-test was used to test differences between 2 groups where 1 of the groups contained continuous variables.

\section{Results}

During the study period from January 1997 through December 2007, 16,540 cranial surgeries had been performed by 25 neurosurgeons. These included elective as well as emergency and trauma cases. Of these procedures, $82(0.5 \%)$ were performed to treat a postoperative infection in a total of 50 patients. The median age was 51 years (range 2-74 years). There were 26 male and 24 female patients. The craniotomies were elective in 40 patients $(80 \%)$. The remaining $10(20 \%)$ were performed on an emergency basis.

Change in mental status was the most common presenting symptom and was present in 18 of 50 patients (Table 1). Other common presenting symptoms were purulent drainage (17), fever (11), headache (10), and swelling (7). Only 2 patients presented with seizures. Many patients had $>1$ symptom at presentation.

Most patients had $>1$ type of postoperative infection (Table 2). Epidural empyema was present in 27 patients, and subdural empyema was present in 7 . There were 8 supratentorial abscesses and 2 infratentorial abscesses. Bone flap removal was necessary in 22 patients who had a bone flap infection. Although wound infections alone were not included in this series, 26 of the 50 patients described above also presented with gross evidence of wound infection. There was no significant association between bone flap removal and the need for multiple operations for infection control $(\mathrm{p}<0.318$, chi-square test).

Twenty-eight patients (56\%) underwent only 1 operation, whereas $15(30 \%)$ patients required 2 operations, and $5(10 \%)$ patients required 3 operations to successfully treat the postoperative infection. Only 2 patients $(4 \%)$ underwent $>4$ operations. The mean number of operations was 1.64 (median 1, range 1-5).

The most common offending organism was methicillinsensitive Staphylococcus aureus, which was found in 10 of 50 patients (Table 3 ). This was in contrast to methicillinresistant $S$. aureus, which was present in only 2 patients. Six patients had coagulase-negative $S$. aureus, 15 had gram-negative rods, 5 had multiple organisms, and 2 patients had a Candida infection. Six patients had negative cultures. 
TABLE 3

Types of organisms causing infection

\begin{tabular}{lc}
\hline \hline \multicolumn{1}{c}{ Organism } & No. of Patients (\%) \\
\hline $\begin{array}{l}\text { Staphylococcus spp. } \\
\text { methicillin-resistant } \\
\text { methicillin-sensitive }\end{array}$ & $2(4)$ \\
coagulase-negative & $10(20)$ \\
gram-negative & $6(12)$ \\
Enterobacter & $3(6)$ \\
Pseudomonas & $4(8)$ \\
Serratia & $2(4)$ \\
other & $6(12)$ \\
Streptococcus spp. & $2(4)$ \\
Propionibacterium spp. & $2(4)$ \\
multiple organisms & $5(10)$ \\
Candida spp. & $2(4)$ \\
culture-negative & $6(12)$ \\
total & $50(100)$ \\
\hline
\end{tabular}

There was no significant association between the type of infectious organism and the need for $>1$ operation for infection control (negative culture $\mathrm{p}<0.168$, polymicrobial infection $\mathrm{p}<0.091$, Staphylococcus spp $\mathrm{p}<0.548$; chi-square test).

Most craniotomies leading to subsequent infection were performed for tumors or other mass lesions (23 of 50 patients) (Table 4). Two of these 23 patients had undergone Gliadel Wafer (MGI Pharma) placement at the time of the original surgery. Seven of 50 patients had undergone craniotomy for vascular lesions. Nine cases involved craniotomy for hemorrhage. There were 4 decompressive craniotomies, 1 seizure surgery, and 1 infratentorial craniotomy. Nine of the patients' original surgeries were for traumatic head injury. The remaining 41 patients' original operations were not related to trauma. The median duration between craniotomy and presentation of postoperative infection was 1.5 months (range 4 days -5 years).

Twenty-seven patients underwent only 1 cranial surgery before presenting with postoperative infection. Two previous cranial operations had been performed in 14 patients. Nine patients had $>2$ previous operations.

Patient outcome at time of last follow-up was measured using the Glasgow Outcome Scale. The mean Glasgow Outcome Scale score was 4.22 (median $5 \pm 1.1$ ).

\section{Discussion}

A $0.8-7 \%$ incidence of postoperative infection has been reported in large series of neurosurgical procedures in which patients received preoperative antibiotics prophylactically. ${ }^{15,9,15,19}$ Most of these studies included all postoperative infections including nonoperative cases. Given the wide range of incidence of postoperative infection reported in the literature, we reviewed our experience at the Barrow Neurological Institute, which is one of the highest volume centers in North America. The aim of this retrospective review was to definitively determine the rate of postcraniotomy infections that required reoperation for pus evacuation. We conducted a retrospective review of the postoperative infections after cranial surgery over the past 10 years. We then focused on those patients who required reoperation to control the infection, not including wound revision alone.

In our series, there were 50 such reoperations for postoperative infection of 16,540 cranial operations for an incidence of $0.5 \%$. This is somewhat lower than some of the other large series that have reported $>1000$ intracranial operations. McClelland and $\mathrm{Hall}^{9}$ reported their experience with postoperative central nervous system infection after 1587 elective cranial operations. Excluding 4 cases of meningitis, they reported a postoperative infection in 10 patients $(0.63 \%)$, which is in line with our findings. Nonetheless, there are several key differences between the 2 series that make direct comparison difficult. The series by McClelland and Hall was a single-surgeon series, and all cases were elective. Our experience included multiple surgeons and a significant number of emergency cases. Interestingly, $35 \%$ of their cases of postoperative infection were associated with indwelling catheters, whereas this rate was much lower in our series. The most common offending organism in our series was $S$. aureus, which occurred in $36 \%$ of the cases. This is in line with previous studies that have reported an S. aureus infection rate of up to $50 \%$. $^{2,7-9,20}$

In another large series, Korinek ${ }^{7}$ performed a prospective multicenter study of 2944 adults who underwent craniotomy during a 15-month period. The patients were evaluated for development and risk factors of neurosurgical site infection. Excluding 56 cases of meningitis and 30 cases of wound infection, there were 87 instances (3\%) of postoperative infection. These included 56 cases of meningitis, 14 bone flap infections, and 17 brain abscesses. Independent

TABLE 4

Types of surgery

\begin{tabular}{lccc}
\hline \hline & & No. of Patients (\%) & \\
\cline { 2 - 4 } \multicolumn{1}{c}{ Distribution } & Elective Op & Emergency Op & Total \\
\hline craniotomy for vascular lesion & $7(14)$ & $0(0)$ & $7(14)$ \\
craniotomy for tumor or mass lesion & $23(46)$ & $0(0)$ & $23(46)$ \\
craniotomy for hemorrhage & $1(2)$ & $1(2)$ & $2(4)$ \\
decompressive craniectomy & $2(4)$ & $0(0)$ & $2(4)$ \\
infratentorial craniectomy or craniotomy & $1(2)$ & $1(2)$ & $2(4)$ \\
seizure surgery & $1(2)$ & $0(0)$ & $1(2)$ \\
trauma & $1(2)$ & $6(12)$ & $7(14)$ \\
$\quad$ craniotomy for hemorrhage & $0(0)$ & $2(4)$ & $2(4)$ \\
$\quad$ decompressive craniectomy & $4(8)$ & $0(0)$ & $4(8)$ \\
other & $40(80)$ & $10(20)$ & $50(100)$ \\
total & & & \\
\hline
\end{tabular}


risk factors for infection were CSF leakage and subsequent operation. Independent predictive risk factors were nonelective surgery, clean-contaminated and dirty wounds, operative time $>4$ hours, and more recent neurosurgery. Presence of antibiotic prophylaxis was not a factor. ${ }^{7}$ The $4 \%$ postoperative infection rate again included a significant number of nonoperative infections. We did not find a statistically significant increase in postoperative infection in trauma versus nontrauma patients. This lack of a statistically significant increase may, however, reflect the small number of cases in the series.

The retrospective case series design reflects the rarity of the phenomenon being studied. This design is most useful for defining natural history and incidence. We did not obtain the characteristics of the $>15,000$ patients who underwent surgery for causes other than postcraniotomy infection. Therefore, no meaningful statistical analysis was possible in respect to the entire craniotomy patient population. We did not find any association between the infecting organism and need for multiple operations to clear the infection. This absence of significant association, however, may reflect the small number of cases in the series. Multiple surgeons performed the operations during the 10-year span of the study. This naturally can be a confounding factor. In the present series we did not compare the duration of surgery to the incidence of postoperative infection, although many previous studies have shown a positive correlation between length of surgery and the risk of infection. ${ }^{7}$

Because of the retrospective nature of this study, it is reasonable to assume that a number of patients with postoperative infection were not included if they presented to and were subsequently treated at other facilities. There is no way for us to determine the number of such patients, which could be high. Possible reasons could include patient dissatisfaction with their original hospital stay, patient migration to a different geographic area, or patients presenting to an outside institution through the emergency department, where the neurosurgeon then performs an emergency operation.

It is worth noting that only 22 of 50 patients had bone flap removal at the time of surgery for infection. The most common reason was the assessment at time of surgery that the bone flap was not grossly infected, that is, in cases of deep abscess. There was no statistically significant association between bone flap removal and infection recurrence $(\mathrm{p}<0.318)$.

There was also a lack of uniformity in the administration of prophylactic antibiotics. Although all patients received a dose of antibiotics immediately before surgery, some patients did not receive any routine postoperative antibiotics. Others were placed on a 24-hour postoperative antibiotic regimen. Although available literature has not shown a definite benefit for postoperative antibiotic prophylaxis, this may or may not be a confounding factor in our series.

\section{Conclusions}

Postoperative infection after cranial surgery is an important phenomenon that needs immediate recognition. Even with strict adherence to sterile technique and administration of antibiotic prophylaxis, a small percentage of patients still go on to develop infection severe enough to require reoperation for significant pus evacuation.

\section{References}

1. Alazia M, Bruder N: [Antibiotic prophylaxis in craniocerebral wounds.] Ann Fr Anesth Reanim 11:705-710, 1992 (Fr)

2. Apisarnthanarak A, Jones M, Waterman BM, Carroll CM, Bernardi R, Fraser VJ: Risk factors for spinal surgical-site infections in a community hospital: a case-control study. Infect Control Hosp Epidemiol 24:31-36, 2003

3. Benca J, Ondrusova A, Kisac P, Krcmery V: Postoperative meningitis: shift in etiology? Neuro Endocrinol Lett 28 (3 Suppl):22, 2007

4. Djindjian M, Lepresle E, Homs JB: Antibiotic prophylaxis during prolonged clean neurosurgery. Results of a randomized doubleblind study using oxacillin. J Neurosurg 73:383-386, 1990

5. Gaillard T, Gilsbach JM: Intra-operative antibiotic prophylaxis in neurosurgery. A prospective, randomized, controlled study on cefotiam. Acta Neurochir (Wien) 113:103-109, 1991

6. Hlavin ML, Kaminski HJ, Fenstermaker RA, White RJ: Intracranial suppuration: a modern decade of postoperative subdural empyema and epidural abscess. Neurosurgery 34:974-981, 1994

7. Korinek AM: Risk factors for neurosurgical site infections after craniotomy: a prospective multicenter study of 2944 patients. The French Study Group of Neurosurgical Infections, the SEHP, and the C-CLIN Paris-Nord. Service Epidémiologie Hygiène et Prévention. Neurosurgery 41:1073-1081, 1997

8. Korinek AM, Baugnon T, Golmard JL, van Effenterre R, Coriat P, Puybasset L: Risk factors for adult nosocomial meningitis after craniotomy: role of antibiotic prophylaxis. Neurosurgery 59: 126-133, 2006

9. McClelland S III, Hall WA: Postoperative central nervous system infection: incidence and associated factors in 2111 neurosurgical procedures. Clin Infect Dis 45:55-59, 2007

10. Mindermann T, Zimmerli W, Gratzl O: Randomized placebo-controlled trial of single-dose antibiotic prophylaxis with fusidic acid in neurosurgery. Acta Neurochir (Wien) 121:9-11, 1993

11. Nathoo N, Nadvi SS, van Dellen JR: Cranial extradural empyema in the era of computed tomography: a review of 82 cases. Neurosurgery 44:748-754, 1999

12. Nathoo N, Nadvi SS, van Dellen JR, Gouws E: Intracranial subdural empyemas in the era of computed tomography: a review of 699 cases. Neurosurgery 44:529-536, 1999

13. Nazzaro JM, Craven DE: Successful treatment of postoperative meningitis due to Haemophilus influenzae without removal of an expanded polytetrafluoroethylene dural graft. Clin Infect Dis 26: 516-518, 1998

14. Post EM, Modesti LM: "Subacute" postoperative subdural empyema. J Neurosurg 55:761-765, 1981

15. Rocca B, Mallet MN, Scemama F, Malca S, Chevalier A, Gouin F: [Perioperative remote infections in neurosurgery. Role of antibiotic prophylaxis.] Presse Med 21:2037-2040, 1992 (Fr)

16. Rousseau JM, Soullie B, Villevielle T, Koeck JL: Efficiency of cefepime in postoperative meningitis attributable to Enterobacter aerogenes. J Trauma 50:971, 2001

17. Rousseaux M, Lesoin F, Clarisse J, Lozes G, Jomin M: [Postoperative abscesses and empyemas. Apropos of 13 cases.] Neurochirurgie 32:304-310, 1986 (Fr)

18. Shapiro M, Wald U, Simchen E, Pomeranz S, Zagzag D, Michowiz SD, et al: Randomized clinical trial of intra-operative antimicrobial prophylaxis of infection after neurosurgical procedures. J Hosp Infect 8:283-295, 1986

19. Tenney JH, Vlahov D, Salcman M, Ducker TB: Wide variation in risk of wound infection following clean neurosurgery. Implications for perioperative antibiotic prophylaxis. J Neurosurg 62: 243-247, 1985

20. van Ek B, Bakker FP, van Dulken H, Dijkmans BA: Infections after craniotomy: a retrospective study. J Infect 12:105-109, 1986

21. Vogelsang JP, Wehe A, Markakis E: Postoperative intracranial abscess - clinical aspects in the differential diagnosis to early 


\section{Operative intracranial infection following craniotomy}

recurrence of malignant glioma. Clin Neurol Neurosurg 100: $11-14,1998$

22. Winkler D, Rehn H, Freckmann N, Nowak G, Herrmann HD: Clinical efficacy of perioperative antimicrobial prophylaxis in neurosurgery-a prospective randomized study involving 159 patients. Chemotherapy 35:304-312, 1989

23. Young RF, Lawner PM: Perioperative antibiotic prophylaxis for prevention of postoperative neurosurgical infections. A randomized clinical trial. J Neurosurg 66:701-705, 1987

24. Zhu XL, Wong WK, Yeung WM, Mo P, Tsang CS, Pang KH, et al: A randomized, double-blind comparison of ampicillin/sulbactam and ceftriaxone in the prevention of surgical-site infections after neurosurgery. Clin Ther 23:1281-1291, 2001

Manuscript submitted January 10, 2008.

Accepted March 11, 2008.

Address correspondence to: Nicholas Bambakidis, M.D., 2910 North Third Avenue, Phoenix, Arizona 85013. email: nicholas. bambakidis@bnaneuro.net. 